\title{
Cover slip external cavity diode laser
}

\author{
Adra V. Carr, Yancey H. Sechrest, Scott R. Waitukaitis, John \\ D. Perreault ${ }^{a}$, Vincent P.A. Lonij and Alexander D. Cronin \\ University of Arizona, Department of Physics, Tucson, AZ, 85721 and \\ ${ }^{a}$ University of Colorado, JILA, Boulder, CO 80309-0440
}

(Dated: July 26, 2007)

\begin{abstract}
The design of a $671 \mathrm{~nm}$ diode laser with a mode-hop-free tuning range of $40 \mathrm{GHz}$ is described. This long tuning range is achieved by simultaneously ramping the external cavity length with the laser injection current. The external cavity consists of a microscope cover slip mounted on piezoelectric actuators. In such a configuration the laser output pointing remains fixed, independent of its frequency. Using a diode with an output power of 5-7 $\mathrm{mW}$, the laser linewidth was found to be smaller than $30 \mathrm{MHz}$. This cover slip cavity and feedforward laser current control system is simple, economical, robust, and easy to use for spectroscopy, as we demonstrate with lithium vapor and lithium atom beam experiments.
\end{abstract}

External cavities are often used to control the frequency of a laser. Early investigations with a plane mirror reflecting light back into a laser (the design in figure 1) showed that optical feedback can "pull" the laser frequency and influence which laser-cavity mode is active 1, 2]. Instead of a mirror a reflective grating can be used, as in the popular Littrow $[\underline{3}, 4,45,6,6,6$, 10] or Littman-Metcalf [10, 14, 15, 16] configurations with diode lasers. However, scanning the cavity alone offers a limited mode-hop-free tuning range with red diode lasers. This was reported by [5, 8, 10], and confirmed in our laboratory. We found that $2 \mathrm{GHz}$ was the maximum single-mode tuning range we could achieve when using only optical feedback to control the frequency of a $671 \mathrm{~nm}$ diode laser. We tried seven different diode laser models [9], and found the same limited tuning range with either a grating or a mirror forming the external cavity. Adjusting only the diode laser current also resulted in a limited $(2-3 \mathrm{GHz})$ mode-hop-free tuning range. This scan range is limited by the overlap of the modes for the laser diode and external cavity. A substantial increase in the tuning range was caused by modulating both the external cavity and the diode laser current together.

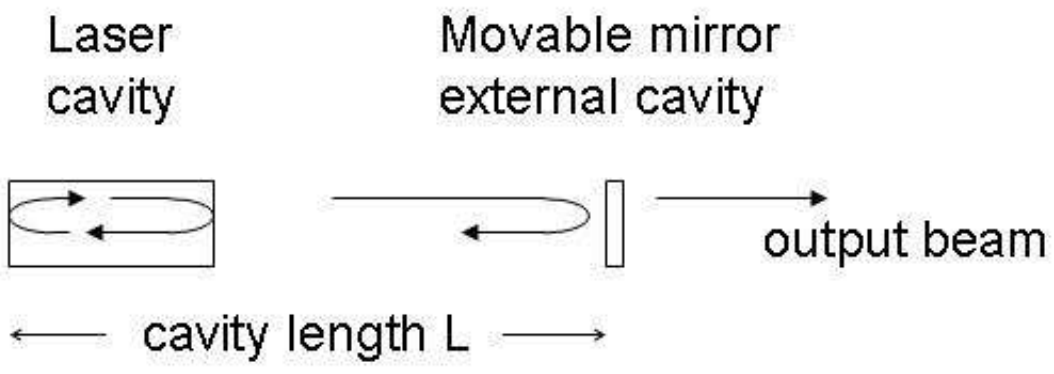

FIG. 1: The external cavity. A movable mirror reflects some light back to the laser. This builds up the power for circulating light with a wavelength that is resonant with both the laser cavity and the external cavity.

Here we describe a diode laser system in which the laser current and the external cavity length are simultaneously ramped to achieve a $40 \mathrm{GHz}$ mode-hop-free tuning range operating near the lithium atom 2S-2P transition wavelength of $671 \mathrm{~nm}$. A microscope cover slip mounted on a piezoelectric transducer serves as the external cavity and output coupler, hence we refer to this as a cover slip cavity [17]. Surprisingly, the modest reflectivity of the glass cover slip surface with no coating $(\mathrm{R} \approx 4 \%)$ is sufficient to force single-mode operation while the laser frequency scans continuously over $40 \mathrm{GHz}$. Since either end of this mode-hop-free tuning range can be extended by adding (or subtracting) an offset to the cavity length, this mode-hop-free tuning range appears to be limited by non-linearity in the required diode laser current or cavity length adjustments.

It was found empirically that the laser current affects the frequency with the slope $d I / d \nu=-250 \mu \mathrm{A} / \mathrm{GHz}$ for the Sanyo model DL3149-057 diode laser. The cavity length affects the frequency with the slope $d L / d \nu=-22 \mathrm{~nm} / \mathrm{GHz}$. This is consistent with the relationship $\Delta L / L=-\Delta \nu / \nu$ for our cavity (length $L=10 \mathrm{~mm}$ ) and red light $(\nu=447 \mathrm{THz}$ ). Therefore, as the cavity length increases by $22 \mathrm{~nm}$, the laser current should be increased by $250 \mu \mathrm{A}$ to anticipate the $-1 \mathrm{GHz}$ shift in laser frequency. This "feedforward" procedure increases the tuning range of the cover slip cavity laser. As a feedforward system, there is no feedback loop connecting the laser current and cavity length adjustments; 

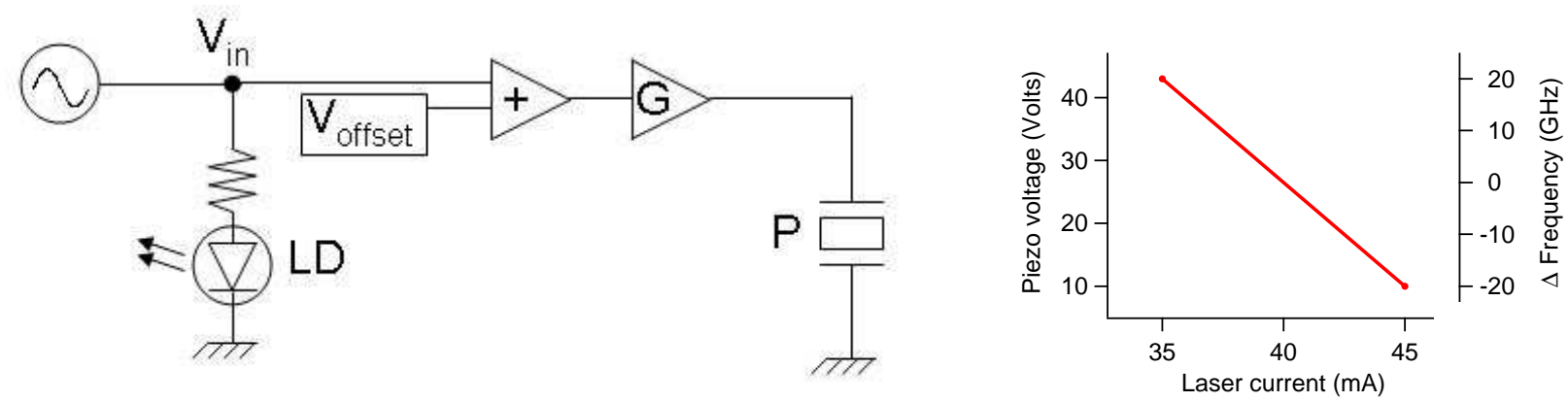

FIG. 2: (Left) Feedforward electronics. Current through the laser diode (LD) depends on the input voltage $\left(V_{i n}\right)$. The same voltage is shifted (by adding $V_{\text {off set }}$ ) then amplified by gain $(\mathrm{G})$ and applied to the piezoelectric actuator (P). Not shown: laser protection circuitry. (Right) The predicted linear relation between the laser current and the cavity actuator voltage and the resulting shift in laser frequency. (The laser frequency is centered on 447,000 GHz for the wavelength $671 \mathrm{~nm}$ ). Nonlinearities in the laser current as a function of $V_{i n}$, nonlinearities in the piezo-voltage as a function of $V_{i n}$ or nonlinearities in the relationship of these quantities to laser frequency limit the mode-hop-free scan range of the system.

they are just commensurately scanned in a predictable fashion using analog electronics. The temperature of the laser mounting block was also stabilized to within $0.1{ }^{\circ} \mathrm{C}$ because temperature was seen to affect the laser frequency with the slope $d T / d \nu=-0.03^{\circ} \mathrm{C} / \mathrm{GHz}$ (i.e. a wavelength shift of $1 \mathrm{~nm}$ per $20^{\circ} \mathrm{C}$ ).

The laser current is modulated by an input voltage $V_{i n}$ that can be provided by a function generator. A resistor in series with the laser keeps the current linearly related to the input voltage $\left[I=\left(V_{\text {in }}-V_{\text {diode }}\right) / R\right.$ to first order above the diode knee]. The same input voltage is also used to control the cavity length. To accomplish this, $V_{i n}$ is added to an offset $\left(V_{\text {offset }}\right)$, amplified with a gain factor $G$, and then directly applied to the piezoelectric actuators to adjust the cover slip cavity length. Hence the cavity length $\left[L=L_{0}+\left(V_{i n}+V_{o f f s e t}\right) G(d L / d V)\right]$ is also linearly related to the input voltage. The length-to-voltage multiplier $(d L / d V=4 \mu \mathrm{m} / 150 \mathrm{~V})$ of the piezoelectric actuators [11] makes the required piezo-voltage to laser current slope $-3.3 \mathrm{~V} / \mathrm{mA}$ for mode-hop-free laser scanning as illustrated in Figure 2.

Ramping the laser current simultaneously with the cavity length is not a novel idea. This was also used to enhance the tuning range for the various diode laser systems which utilized an external cavity grating [3, 4, 5, 6]. However, our cover slip cavity with feedforward current control has the advantages of simplicity, economy, and stability. We discuss these advantages after explaining the mechanical construction.

Mechanical views of the cover slip cavity, collimating optics, and temperature-stabilized laser mount are shown in Figure 3. The physical layout has two principle parts: a mounting block, and a kinematic mount. The laser diode is mounted in a 0.6 "-diameter tube with collimating optics [12] and the tube is clamped in a 2 " $\times 1$ " $x 1$ " aluminum block pictured in Figure 3. A temperature sensor (AD590) and heaters (a 5 Ohm resistor and 2 transistors) are also mounted on the block.

The stock 1"-square kinematic mount [13] is disassembled and a 0.7 "-square hole is milled in one plate (as shown in Figure 3). A 0.3"-diameter hole is drilled in the other plate to transmit the output beam. Two piezoelectric actuators are mounted on either side of this output hole, and a 0.5 " diameter glass cover slip is attached to the actuators with epoxy. The cover slip is thus positioned within the kinematic mount so that it can form a short external cavity. The kinematic mount is attached to the aluminum block with epoxy so that the cover slip and output hole are centered on the laser beam. The mount enables coarse alignment of the cover slip after the initial construction. During feedforward operation, the system's only moving parts are the cover slip and the piezoelectric actuators. The cavity length can be coarsely adjusted (from $10 \mathrm{~mm}$ to $20 \mathrm{~mm}$ ) by relocating the collimating tube, and with care the kinematic mount can be taken apart (to replace the cover slip) and reassembled while the plate with the $0.7^{\prime \prime}$ hole remains attached to the mounting block.

The block and cavity assembly is mounted on a standard optics post and positioned in a thermally stable enclosure (with a hole for the output beam). To reduce vibrations, the entire system is mounted on a $1 \mathrm{Kg}$ brass plate and placed on top of sorbathane pads.

One advantage of the cover-slip cavity is simplicity. If a grating were used for the external cavity there are two mechanical design criteria that must be considered because both the cavity length as well as the grating angle affect the laser frequency. In comparison, our cover slip cavity has only one important dimension: cavity length. The laser beam always points in the same direction with the cover slip cavity (whereas some grating cavities shift the beam direction as the frequency is scanned). The cover slip transmits $\sim 92 \%$ of the laser beam so it offers a high output efficiency. We avoided anti-reflection coating the diode (as was done in [5, 8, 18]), we avoided removing the diode 

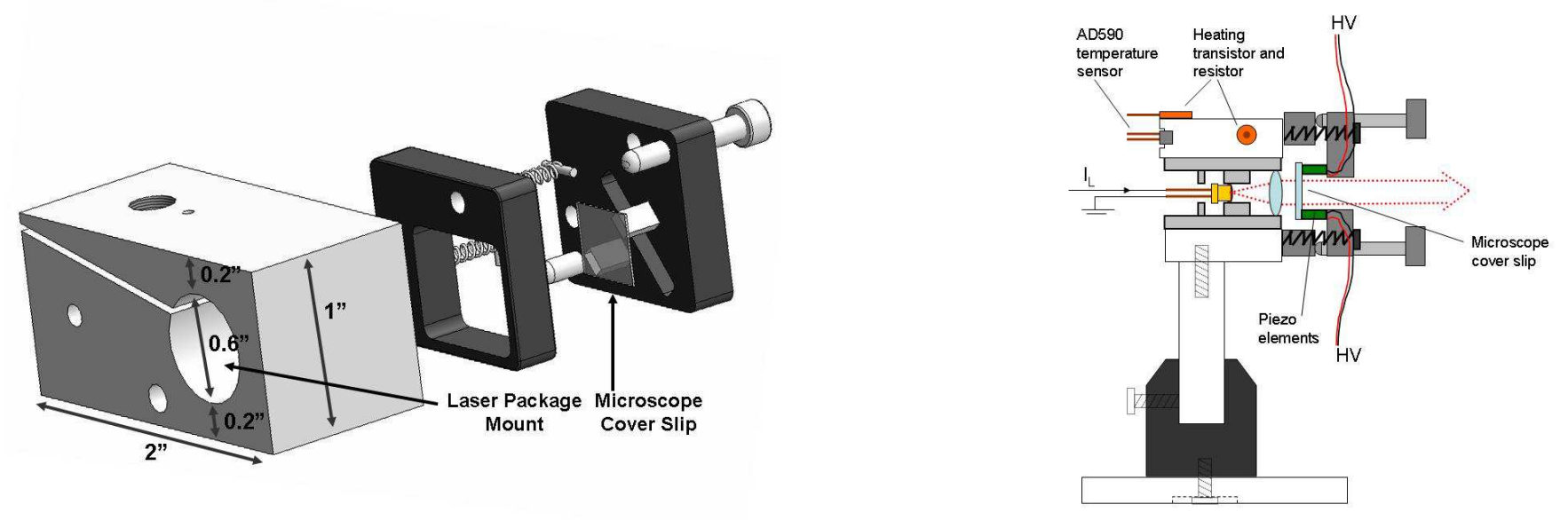

FIG. 3: (Left) Exploded view of the laser mounting block and cover slip cavity assembly. (Right) Side view of the laser assembly.

can (which has glass surfaces) and we avoided coating either side of the cover slip (either to increase or decrease reflectivity) in order to keep the design simple. The entire laser system was constructed from parts that cost $\$ 1500$, and a lithium heat-pipe vapor cell was constructed for a cost of $\$ 800$. The parts are listed in Appendix I.

If the temperature of the mounting block drifts by even $0.1^{\circ} \mathrm{C}$ then the offset voltage $V_{\text {offset }}$ needs to be adjusted, but this is the only intervention needed to keep the system scanning across the lithium atom resonance features for days at a time. This stability and long tuning range makes the red light particularly useful for instructional laboratories and atomic physics research. We operated individual Sanyo DL3149-057 lasers for over 6 months with the current ramping in the range 35 to $45 \mathrm{~mA}$. The laser is typically scanned by applying a triangle waveform to $V_{i n}$ that varies from 8 to 10 volts and cycles at $100 \mathrm{~Hz}$. For some applications we also locked the laser frequency to a saturated absorption feature by connecting $V_{i n}$ to the output of a locking circuit.

A number of experiments were done to test the performance of the cover slip cavity system. Lithium atom absorption spectra demonstrating a 40-GHz mode-hop-free frequency scan are shown in Figure 4. The vapor cell absorption features (Fig 4 upper left) each have a $3 \mathrm{GHz}$ Doppler width due to thermal motion of the lithium atoms. In this plot, data are shown (lower left) for two vapor cell temperatures with correspondingly different optical depths. The flat-bottomed absorption features with the higher optical depth allowed us to measure the amount of off-mode light in the laser output beam. This was seen to be $7 \pm 2 \%$. The laser intensity is modulated from 5 to $7 \mathrm{~mW}$ in accord with the laser current, but the laser beam pointing remains stable during the scan. A Fabry-Perot transmission spectrum is shown in Figure 4 (lower left) confirming the scanning range of $40 \mathrm{GHz}$. Saturated absorption spectra shown in Figure 4 (upper right) were obtained using the two-probe method described in [19, 20]. Also shown is an atom beam deflection signal (bottom right) obtained by shining the cover slip cavity laser beam at a highly collimated atom beam. (See 22] for a description of this technique). Both types of Doppler-free spectra show the large $803 \mathrm{MHz}$ hyperfine splitting due to the $\mathrm{F}=1,2$ levels of the ground $2 S_{1 / 2}$ state of ${ }^{7} \mathrm{Li}$. The atom beam deflection spectrum also shows a smaller but clearly resolved splitting due to the $\mathrm{F}=1,2$ levels in the excited $2 P_{1 / 2}$ state of ${ }^{7} \mathrm{Li}$.

The laser line-width was smaller than $30 \mathrm{MHz}$, as found with a Fabry-Perot interferometer. This upper limit on the linewidth is consistent with the atomic deflection spectra and the saturated absorption spectra shown in figure 4 . In additional tests we modulated the laser current at $800 \mathrm{MHz}$ (to add sidebands to the laser spectrum) as described in [10]. This did not reduce the tuning range. We also used this laser to study Faraday rotation in vapor [24], the Hanle effect in vapor [23], and optical pumping of an atom beam. We thus demonstrated that the cover slip cavity diode laser serves for several atomic physics experiments.

In conclusion, we have described a diode laser system that uses a cover slip cavity and feedforward operation to achieve a mode-hop-free scanning range of $40 \mathrm{GHz}$. The frequency can be scanned over the lithium atom 2S-2P resonances near $671 \mathrm{~nm}$, with the entire system being simple, economical, stable and robust.

\section{Appendix I: Parts and Supplies}

The following is a list of parts used to build the cover slip cavity laser. 

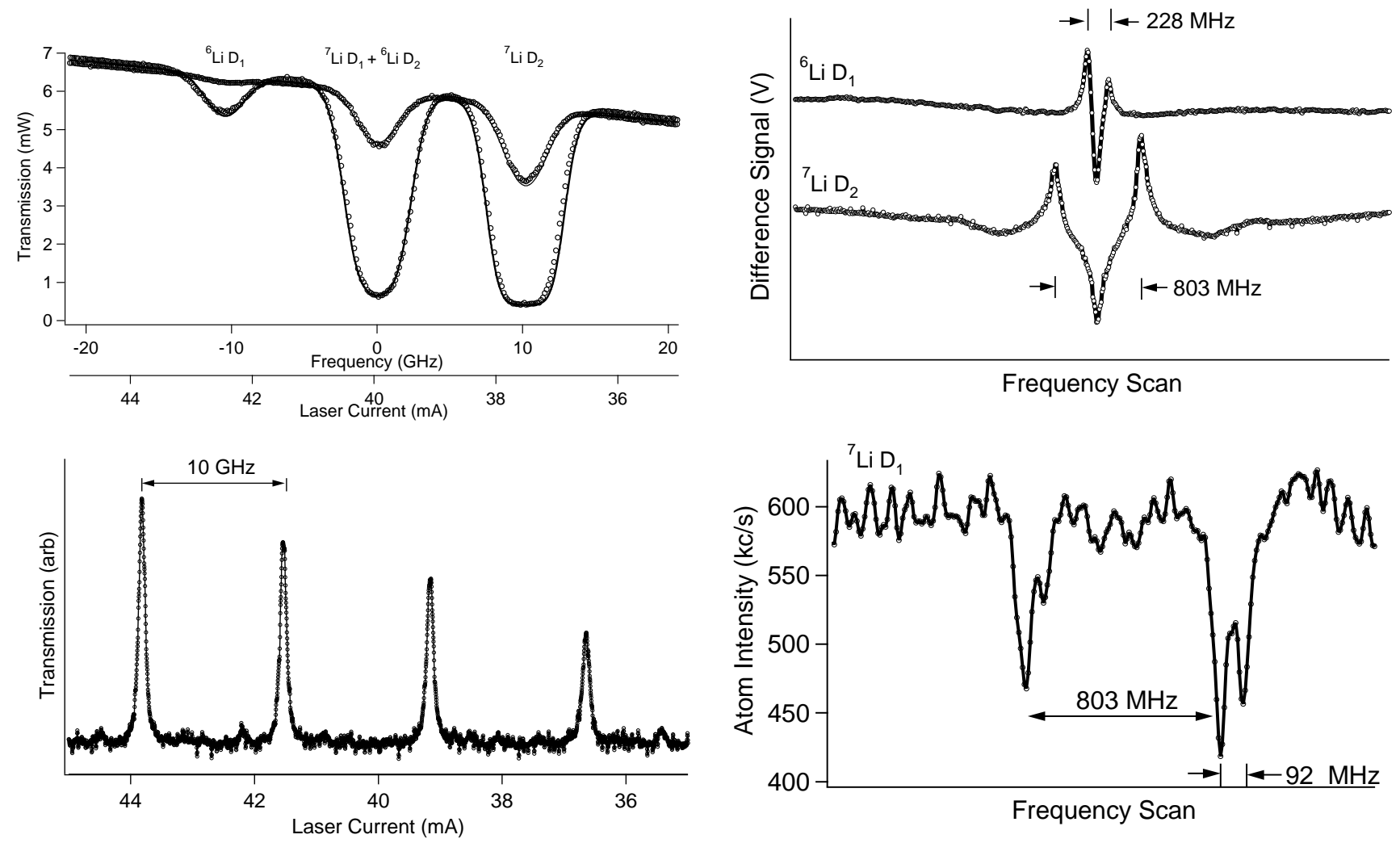

FIG. 4: (Top Left) Atomic transmission spectra from lithium vapor. Two spectra (Top trace: $\mathrm{T}=370{ }^{\circ} \mathrm{C}, \mathrm{Bottom}$ trace: $\mathrm{T}=420^{\circ} \mathrm{C}$ ) demonstrate that higher vapor temperature results in larger optical depth. (Bottom Left) Fabry-Perot transmission spectrum using a Fabry-Perot cavity. (Top Right) Saturated absorption signals. The upward (increased transmission) peaks are due to transitions out of the ground state hyperfine levels. (Bottom Right) Atom beam deflection spectrum. Atom beam count rate is monitored as a function of laser frequency. With the laser beam perpendicular to the atom beam, and the atom beam collimated to $10^{-4}$ radians, the deflection spectrum has essentially zero Doppler width. Power broadening, transit time broadening, and the laser linewidth all contribute to the four observed 55- MHz (FWHM) dips due to deflection. In addition to the $803 \mathrm{MHz}$ spacing due to the ground state hyperfine splitting, the atom beam deflection signal shows the $92 \mathrm{MHz}$ hyperfine splitting due to the $2 P_{1 / 2}$ excited state of ${ }^{7} \mathrm{Li}$.

1. Sanyo Laser Diode: $670 \mathrm{~nm}, 7 \mathrm{~mW}$, DL3149-057, \$14.14, and socket S7060, \$3.98, Thorlabs

2. Collimation Tube with Lens, LT230P-B, \$108.00, and Spannor wrench, SPW301, \$13.00, Thorlabs

3. Kinematic mount, KMS, $\$ 33.50$, Thorlabs

4. Piezoelectric elements (2), AE0203D04, \$72.00, Thorlabs

5. Piezoelectric controller, MDT694A, $\$ 680.00$, Thorlabs

6. Function generator, GFG8020H, \$193.50, Mouser Electronics

7. Sorbathane Pad, SB12B, $\$ 49.00$, Thorlabs

8. Power supply (2), 680-WM113-D5, \$42.20, Mouser Electronics

9. Temperature sensor, AD590, $\$ 12.80$, Thorlabs

10. Thermocouple probe, TJC36CASS020K6SMPM, \$30.00, Omega Inc.

11. Thermocouple controller, HH74K, $\$ 55.00$, Omega Inc. 
Additional stock electronics components that were used to construct the laser (op amps, breadboards, bud boxes, potentiometers), the microscope cover slip, and laboratory supplies such as expoxy all totaling $\$ 120$, are not itemized.

The lithium heat-pipe oven was constructed from the following parts.

1. Optical viewports (2), FVG0150, \$95.26, Varian Inc.

2. 1.5" x 16" Stainless Steel tube w/ CF flanges and Nupro Inc. SS 4BG TSW valve, $\$ 180.00$, custom welded

3. Heater wire, Aerocoax 1HN080B-3.9, $\$ 3.50 / \mathrm{ft}$, ARI Industries Inc.

4. High temp cement, OB600, \$18.00, OMEGA Engineering INC.

5. Variac voltage supply TDGC2-0.5, \$50.00, Circuit Specialists Inc.

6. Fibrafax thermal insulation, 93435K26, \$24.75/ft, McMaster-Carr

7. Thermocouple probe, TJC36CASS020K6SMPM, \$30.00, OMEGA Engineering INC.

8. Thermocouple controller, HH74K, \$55.00, Omega Inc.

9. Li pellets (50g), Alfa Aesar \#41829, \$81.70, Sigma-Aldrich Chemical

The Li heat pipe oven operates with the central region near $400^{\circ} \mathrm{C}$ and tube ends (and viewports) at $80^{\circ} \mathrm{C}$. $\mathrm{Heater}$ wire was wound around the central $5^{\prime \prime}$ of the $16^{\prime \prime}$ long Stainless Steel tube. The central 8" were then covered in 2 " thick fibrafax thermal insulation. The high temperature cement was used to bond the heater wire to standard hookup wire outside the thermal insulation surrounding the oven. Mounts for the heat pipe oven were machined out of stock aluminum. Five grams of Li were loaded in the SS tube, and the assembly was then baked under 50 mT Argon buffer gas at $200^{\circ} \mathrm{C}$ for 2 hours. The oven tube was then sealed with the valve and operated at $400^{\circ} \mathrm{C}$ for over a year.

\section{Appendix II: Control Electronics}

\section{A. Temperature control}

The AD590 temperature sensor produces $1 \mu A / K$. In the circuit shown in Figure 5] the transimpedance gain and offset makes $V_{\text {temp }}$ equal to $0 \mathrm{~V}$ at $20{ }^{\circ} \mathrm{C}$ and $10 \mathrm{~V}$ at $80{ }^{\circ} \mathrm{C}$. The difference between the adjustable $V_{\text {set }}$ and $V_{t e m p}$ makes $V_{\text {err }}$. The error signal $V_{\text {err }}$ is used with feedback to regulate $V_{\text {corr }}$. The correction signal, $V_{\text {corr }}$ is then used to regulate the current supplied to the heating elements on the laser block (shown within dotted line box in Figure 51). Temperature stabilization circuits with similar function are found in [3, 44, 10, 21].

\section{B. Peizo gain and offset}

The piezo elements were controlled with the piezo gain and offset circuit shown in figure 6, This simple circuit was put in line before the high voltage amplifier (piezoelectric controller, MDT694A, Thorlabs).

\section{Appendix III: Initial Alignment Procedure}

These steps are a guide for tuning the cover slip cavity laser frequency to an atomic resonance.

1. Adjust the laser wavelength to within $0.1 \mathrm{~nm}$ of $671 \mathrm{~nm}$ by modifying the mounting block temperature while using a grating spectrometer to monitor the wavelength.

2. Ramp the laser current using a $100 \mathrm{~Hz}$ triangle function. Make the ramp extend from threshold to the maximum safe current (45 mA in the case of the Sanyo model DL3149-057).

3. Tip and tilt the cover slip by using the kinematic mount to provide optical feedback to the laser cavity. Do this first by getting the dim secondary laser beam (caused from the back reflection from the cover slip) to overlap with the primary (brighter) laser beam. 


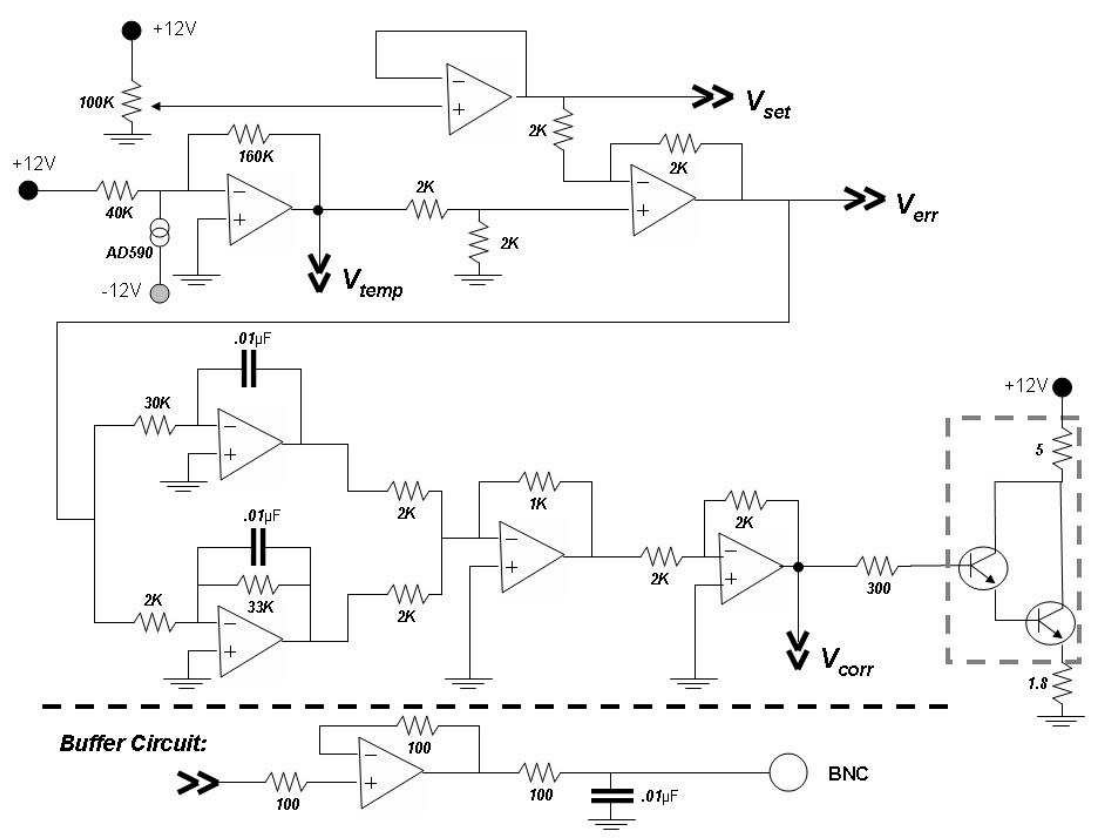

FIG. 5: Temperature control circuit used to heat laser mount block. The $5 \Omega$ resistor and 2 transistors serve as heating elements mounted to the aluminum mounting block. The dashed box indicates which elements are physically mounted onto the aluminum mounting block. The buffer circuit is duplicated four times and utilized where the double-arrows indicate connections to test-points where voltages can be monitored.

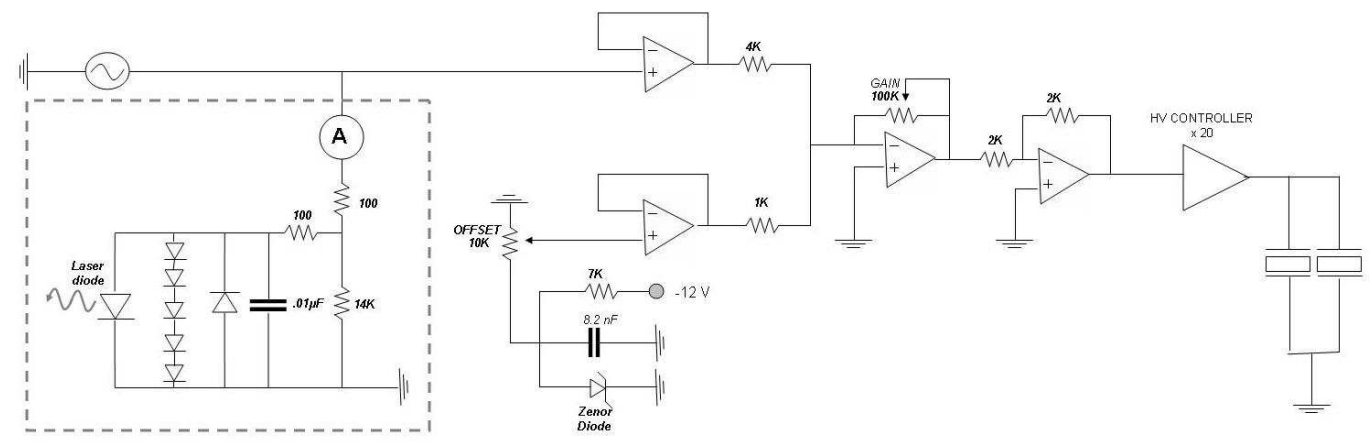

FIG. 6: Feedforward electronics detail. The boxed portion of the circuit shows the laser and its protection circuitry including an ammeter (A). The potentiometers marked "offset" and "gain" serve to condition the voltage applied to the piezoelectric elements.

4. While observing the transmitted power through a low-finesse Fabry-Perot etalon (the windows of the heat-pipe oven can serve this purpose), verify that the piezo-voltage offset and gain can modify the frequencies at which mode-hops occur. Re-align the cover slip using control of the laser modes as an indication of good coupling back into the laser cavity.

5. Observe the transmission through the heat-pipe oven with the laser current driven by the $100 \mathrm{~Hz}$ ramp. Slowly ramp the piezo voltage (with the potentiometer) to deliberately hop between different modes, and scan the temperature in order to see any hint of an atomic absorption (or fluorescence) signal.

6. Once any atomic absorption (or fluorescence) has been observed, then obtain a larger mode-hop-free range by following these steps: (1) adjust the piezo-voltage gain to suppress mode hops, and (2) adjust the piezo-voltage offset to select the laser mode resonant with the atoms.

7. For the largest mode-hop-free tuning range it is often required to iterate steps 1 and 2 and fine tune the laser temperature and the cover slip alignment. 


\section{Acknowledgements}

This work was supported with NSF Grant No. PHY-0653623. We thank Dr. W. Bickel and Dr. J. Jones for diagnostic equipment, K. Guerin for assistance with mechanical drawings, and M. Parker of Rincon Research Inc. for optics components.

[1] R. P. Salathe, Applied Physics 20, 1-18 (1979).

[2] L. A. Coldren and T. L. Koch, Journal of Lightwave Technology 2, 1045-1051 (1984).

[3] K. B. Macadam, A. Steinbach, and C. Wieman, American Journal of Physics 60, 1098-1111 (1992).

[4] L. Ricci, M. Weidemuller, T. Esslinger, A. Hemmerich, C. Zimmermann, V. Vuletic, W. Konig, and T. W. Hansch, Optics Communications 117, 541-549 (1995).

[5] R. S. Conroy, A. Carleton, and K. Dholakia, Journal of Modern Optics 46, 1787-1791 (1999).

[6] A. Andalkar, S. K. Lamoreaux, and R. B. Warrington, Review of Scientific Instruments 71, 4029-4031 (2000).

[7] H. Patrick and C. E. Wieman, Review of Scientific Instruments 62, 2593-2595 (1991).

[8] M. G. Boshier, D. Berkeland, E. A. Hinds, and V. Sandoghdar, Optics Communications 85, 355-359 (1991).

[9] The Sanyo DL3149-057, the Mitsubishi ML101-J8 and the Missubishi ML101-J27 each demonstrated resonant absorption by Li vapor. The Sanyo 4039-11 and Hitachi models HL6720, HL6714G, and HL6724MG were also tested but did not demonstrate resonant absorption by $\mathrm{Li}$ vapor due to unsufficient wavelength tuning range. At least 3 units of each model were tested over a range of temperature from $0^{\circ} \mathrm{C}$ to $80^{\circ} \mathrm{C}$. The Sanyo DL3149-057 gave the largest mode-hop-free tuning range, so results from this model are discussed here.

[10] C. E. Wieman and L. Hollberg, Review of Scientific Instruments 62, 1-20 (1991).

[11] Thorlabs AE0203D04

[12] Thorlabs model LT230P-B

[13] Thorlabs KMS

[14] S. Stry, S. Thelen, J. Sacher, D. Halmer, P. Hering, and M. Murtz, Applied Physics B-Lasers and Optics 85, 365-374 (2006).

[15] K. C. Harvey and C. J. Myatt, Optics Letters 16, 910-912 (1991).

[16] M. G. Littman and H. J. Metcalf, Applied Optics 17, 2224-2227 (1978).

[17] This name was suggested by Dr. Harold Metcalf who built a similar design. John Noe, personal communication, 2007.

[18] K. G. Libbrecht, R. A. Boyd, P. A. Willems, T. L. Gustavson, and D. K. Kim, American Journal of Physics 63, 729-737 (1995).

[19] I. E. Olivares, A. E. Duarte, T. Lokajczyk, A. Dinklage, and F. J. Duarte, Journal of the Optical Society of America B-Optical Physics 15, 1932-1939 (1998).

[20] G. N. Rao, M. N. Reddy, and E. Hecht, American Journal of Physics 66, 702-712 (1998).

[21] J. C. Camparo, Contemporary Physics 26, 443-477 (1985).

[22] A. Askin, Science 210, 1081-1088 (1980).

[23] Alfred Kastler, Nuclear Instruments and Methods 110, 259 (1973).

[24] D.A. Van Baak, Am. J. Phys. 64 (1996). 